\title{
MANTENIMIENTO INDUSTRIAL EN MÁQUINAS HERRAMIENTAS POR MEDIO DE AMFE
}

\section{INDUSTRIAL MAINTENANCE IN MACHINE TOOLS BY MEANS OF AMFE}

\author{
Jesús Vicente González Sosa ${ }^{1, *}$ Jesús Loyo Quijada², \\ Miguel Ángel López Ontiveros ${ }^{3}$, Pedro Pérez Montoya ${ }^{4}$, \\ Alfredo Cruz Hernández ${ }^{4}$
}

\section{RESUMEN}

El mantenimiento industrial se ha utilizado en todos los sectores académicos e industriales dentro de un plan de mantenimiento, lo que permitió fortalecer el entorno de trabajo para obtener resultados en la aplicación de este mismo, por ello es que constantemente se buscan alternativas para la evaluación de éste como parte del desarrollo y éxito en los departamentos correspondientes. La técnica que se utilizó se conoce como Análisis de Modo y Efecto de Fallas (AMFE), cuyo propósito radica en mejorar los rangos de tiempo en el mantenimiento a través de situaciones críticas de un producto o sistema, justificando las mejoras e involucrando a la mayor cantidad de personas que interactúan dentro de un área industrial en conjunto con mantenimiento. Como parte de los resultados, se logró identificar las partes o secciones que recuren constantemente a mantenimiento correctivo, buscando implementar mantenimientos preventivos y predictivos para mejorar las condiciones o ciclo de vida de una máquina herramienta. Se concluye que el efecto de haber aplicado un AMFE a una máquina herramienta para el desarrollo de un producto o una serie de productos fortaleciendo las tendencias y beneficios que ofrece la herramienta en función de reducir tiempos, reducir fases en el mantenimiento, incrementando la fiabilidad y seguridad del equipo evaluado en los procedimientos del mantenimiento industrial, generando nuevas alternativas para la implementación en programas de mantenimiento.

Palabras Clave: Análisis de mantenimiento, análisis modal de fallos y efecto, evaluación de maquinaria, maquinado convencional.

\footnotetext{
${ }^{1}$ Profesor Departamento de Sistemas de la División de Ciencias Básicas e Ingeniería perteneciente a la Universidad Autónoma Metropolitana Unidad Azcapotzalco, Ciudad de México, México. orcid.org/0000-0002-1325-0266

2Profesor Departamento de Sistemas de la División de Ciencias Básicas e Ingenieria perteneciente a la Universidad Autónoma Metropolitana Unidad Azcapotzalco, Ciudad de México, México. orcid.org/0000-0002-2713-723X

${ }^{3}$ Profesor Departamento de Sistemas de la División de Ciencias Básicas e Ingeniería perteneciente a la Universidad Autónoma Metropolitana Unidad Azcapotzalco, Ciudad de México, México. orcid.org/0000-0001-9377-9639

${ }^{4}$ Ingeniería Industrial, División de Ciencias Básicas e Ingeniería, Universidad Autónoma Metropolitana, Unidad Azcapotzalco, Ciudad de México, México.
}

-Autor para correspondencia: jvgs@azc.uam.mx.com 


\section{ABSTRACT}

Industrial maintenance is used in all academic and industrial sectors within a maintenance plan, which allows strengthening the working environment to obtain results in the application of this same, so it is constantly sought Alternatives for the evaluation of this one as part of the development and success in the corresponding departments. The technique to use is known as analysis of mode and fault effect (AMFE), which aims to improve the time ranges in maintenance through critical situations of a product or system, justifying improvements and involving the largest number of people who interact within an industrial area in conjunction with maintenance. As part of the results, it is able to identify those parts or sections that constantly cure corrective maintenance, seeking to implement preventive and predictive maintenance to improve the conditions or life cycle of a machine tool. Finally, it should be noted that the effect of applying an AMFE to a machine tool for the development of a product or a series of products strengthens the tendencies in the procedures of industrial maintenance, encouraging new alternatives of implementation In the maintenance programs.

Keywords: Maintenance analysis, failure modal and effect analysis, machinery evaluation, conventional machining.

\section{INTRODUCCIÓN}

En la actualidad el mantenimiento industrial forma parte esencial en los procesos productivos, dado que tiende a minimizar u optimizar el desarrollo en cuestión. Aunado a ello se deben disminuir las fallas y averías en los sistemas involucrados con el mantenimiento y propiamente en la producción, que se refleja en costos e ingresos para la industria, por lo cual es indispensable la gestión de mantenimiento con base en los procesos industriales (Ortiz-Useche, 2013).

Se hacen uso de diversos métodos para analizar el mantenimiento, entre los más conocidos se tiene el mantenimiento centrado en la confiabilidad (RCM) (Fornés, 2016), y el mantenimiento productivo total (TPM) (Hernández-Gomez et al., 2015), por otro lado el método de análisis de modo y efecto de fallas identifica de manera general las tendencias con las cuales se establecen las mejoras, sin olvidar la participación activa de cada uno de los sectores que intervienen en un proceso de mantenimiento.

El análisis de riesgos es una herramienta que en los procesos de manufactura se considera limitado, por lo que es importante resaltar que para los procesos mencionados la metodología de AMFE (Análisis de Modo y Efecto de Fallas) es la más conocida en ese campo de aplicación, aportando ventajas sustanciales en su desarrollo (Cartín, 2014). Además, estos métodos se establecen como una herramienta válida para generar rendimientos sustanciales con respecto a fallas y averías, optimizándolas para mejorar resultados en la aplicación de ello (Fornés, 2016).

El mantenimiento es una estrategia de etapas y/o actividades que conjuntan el estado óptimo de un servicio, sistema o equipo para mejorar sustancialmente los procesos, con lo cual se enfatiza la calidad como táctica en el desarrollo aplicable para la gestión de mantenimiento, de acuerdo con la naturaleza de este mismo (Pineda-Zapata, 2013). 
El mantenimiento se plantea de manera operativa, como un proceso vinculado con los sistemas administrativo de organizaciones e industrias, logrando con ello la concepción en el uso de AMFE (Hernández-Gomez et al., 2015), en donde se identifican de manera integral el mantenimiento en los sectores industriales y académicos.

En la evaluación de escenarios, se identifica la herramienta análisis de riesgos que impacta de forma directa en un proceso o planta industrial, logrando con ello minimizar los parámetros que identifica el análisis (Vázquez, 2018). Además, las acciones recomendadas que surgen del análisis de riegos generalmente no se implementan, debido al seguimiento que debe realizarse de forma constante (COMIMSA, 2008). Y en la actualidad se busca identificar nuevas técnicas para aumentar la eficiencia en los planes de mantenimiento y la gestión.

En el vínculo de servicios, existen interacciones con el AMFE, que muestran e identifican los criterios de fiabilidad para los sectores hospitalarios, en donde se analiza, verifica, evalúa y fomenta la prevención de fallas en los sistemas, incrementado la seguridad en ellos (Mateus, 2015) o en el de informática (Gorbenkoet al., 2005). En este caso, el AMFE permitió identificar parámetros puntuales de la ingeniería, dando como resultado la mejora continua de los sistemas reorganizando de forma correcta el servicio y su planeación como tal, aumentando la satisfacción del cliente (Ebrahimipour et al., 2010).

Existen en la actualidad herramientas que permiten analizar desde enfoques teóricos como prácticos el mantenimiento, utilizar el más adecuado depende de las condiciones y acciones que se pueden llevar a cabo en el área de trabajo y con las personas involucradas en la mejora continua del mantenimiento y los enlaces que se generan con ello, como lo es la producción. La herramienta consiste en recolectar datos como la observación directa, entrevistas no estructuradas y el método de mantenimiento de análisis de modo y efecto de falla (AMFE) para encontrar severidad de fallas y planear acciones de contención para disminuir las fallas en los equipos. Se determinan tareas específicas de mantenimiento a la maquinaria, y medidas de orden y limpieza con la finalidad de realizar un mantenimiento de forma adecuada. (Rosales, 2015). En ese sentido, la importancia de hacer uso de diversos métodos para la fundamentación del mantenimiento es la aplicación del AMFE, como se presenta en este caso en donde se analizas máquinas herramientas de un taller de manufactura, con la finalidad de mejorar las condiciones actuales para el aprovechamiento de este equipo en la etapa de producción. Las técnicas o métodos para la identificación o análisis de riesgos se clasifican en métodos comparativos y métodos fundamentales; el primero se basa principalmente en el conocimiento adquirido con base en la experiencia, listas de verificación, análisis histórico de accidentes y el segundo son formas estructuradas que ayudan a estimular el conocimiento en conjunto, algunos de los métodos son: estudios de riesgo y operatividad, análisis de tareas y análisis preliminar de riesgos. (Herrera- Galan, 2017).

Las consideraciones que se mencionan, dan pauta a realizar un análisis con las características de manifestar la mejora en la gestión y desarrollo del mantenimiento industrial para una mayor formación de los representantes en este ámbito.

\section{Mantenimiento}

Los tipos de mantenimiento son:

a) Correctivo: también conocido como reactivo, se enfoca en llevar a cabo acciones correctivas en un equipo o sistema una vez que éste ha fallado, se aplica en detección 
total, parcial y mínima, logrando asimismo disminución de horas productivas, la principal característica radica en aplicarse cuando ocurre una falla y los gastos son impredecibles.

b) Preventivo: conocido con el nombre de planificado, se basa en considerar acciones denominadas correctivas en periodos fijos durante el proceso de producción, de acuerdo a las recomendaciones elaboradas por los fabricantes y la experiencia de los operadores de equipo. Se llevan a cabo inspecciones en todos los sistemas de los equipos a través de los mecánicos y administradores de mantenimiento.

c) Predictivo: en este nivel se utiliza la tecnología de vanguardia en efectos de mantenimiento con los que se logra medir parámetros en los equipos, dando un indicativo para el tipo de falla que se pudiese presentar en el sistema. $Y$ como resultado de dicho análisis se toman acciones correctivas en el momento que el parámetro alcance valores críticos.

d) Proactivo: este tipo de mantenimiento pretende sensibilizar en todo momento a todos los departamentos de la planta para asegurar que el proceso se lleva a cabo de manera correcta en colaboración con los encargados correspondientes, además, ofrece un impacto en lo económico logrando disminuir en lo posible los paros de producción no programados, dando solución a la problemática que se pudiera presentar. Todo ello debe generarse por medio de un reporte del progreso y actividades desarrolladas.

\section{Análisis de Modo y Efecto de Fallas (AMFE)}

AMFE, tiene como objetivo analizar los problemas potenciales que se consideran y abordan a través de procesos de desarrollo del producto, considerando que es una metodología analítica (Napoles-Villa, 2016, García, 2012).

AMFE puede ser utilizado en a) sistema, b) diseño y c) proceso, antes, durante y después de la acción a ejecutar. En la Figura 1 se tiene una representación del AMFE y los casos de aplicación.

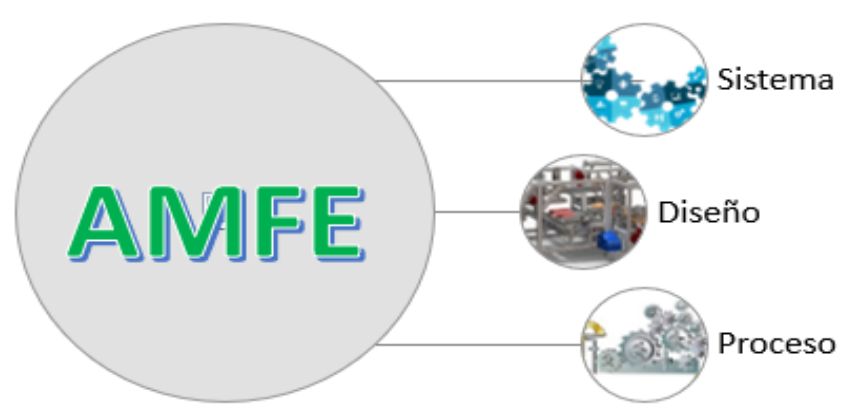

Figura 1. Aplicaciones del AMFE

Para un mejor manejo del mantenimiento, es realizar AMFE desde la parte de diseño, y en ese sentido el enfoque dará mayores resultados en su aplicación y aprovechamiento como metodología a implementar dentro de la gestión del mantenimiento en los sectores industriales. Sin embargo, no se debe olvidar que la industria generalmente utiliza o lleva a 
cabo el AMFE de sistema y proceso, dadas las condiciones de existencia de equipos y planta. Dos elementos fundamentales para la aplicación del AMFE se encuentran fusionados de tal manera que logran ejercer programas de mantenimiento funcionales, los elementos son el tiempo y la oportunidad de implementar la herramienta en el entorno deseado, logrando con ello acciones previas dentro del sistema evaluado. Asimismo, se plantea que el impacto del AMFE persevera cuando la implantación del análisis de fallas optimiza el proceso, producto o sistema.

\section{MATERIAL Y MÉTODOS}

\section{Metodología de análisis}

El proceso para la aplicación de la metodología de análisis consistió:

a) Se seleccionó el equipo: máquinas herramientas conocidas como torno, fresadora y taladro industrial, considerándolas a cada una como estudio de caso.

b) Se obtuvo la especificación técnica: en donde se establecen los parámetros para trabajar, analizar, desarrollar y aplicar las mejoras en cada caso.

c) Se identificaron las partes que componen al sistema o máquina de estudio.

d) Se identificaron responsables, supervisores, técnicos, gerentes, mantenimiento que participan en la gestión.

e) Se aplicó AMFE.

El proceso para dicho análisis es de manera cíclica, el cual se aprecia en la figura 2, logrando con ello identificar los pasos de la metodología en cuestión para realizar el análisis.

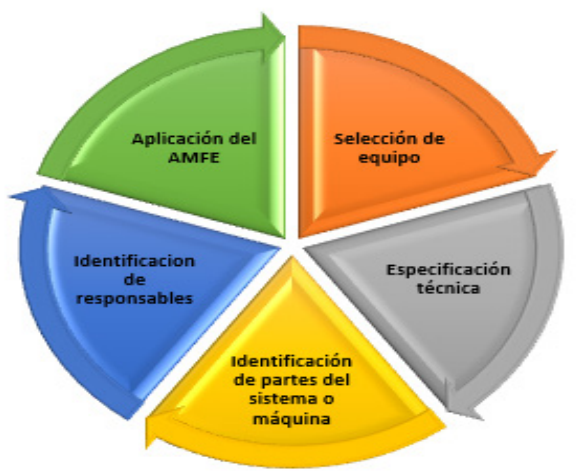

Figura 2. Proceso para analizar el mantenimiento por medio de AMFE

La Figura 2 muestra la sinergia que debe existir en las etapas que conforman el método en la realización para el AMFE en un equipo determinado, considerándolo de esta manera, cíclico, debido a que se culmina una etapa y debes seguir la secuencia hasta lograr el resultado esperado, iterando las veces necesarias, las cuales dependerán de la experiencia en los equipos y máquinas sobre las cuales se lleva a cabo el análisis (Consuegra, 2015). Por otro lado, el proceso se desarrolla de esta manera con la finalidad de mantener la claridad en la secuencia de cada uno de los pasos y lograr el objetivo esperado en el AMFE, tomando en cuenta los criterios de producción en cada máquina (González, 2017). 
En el diagrama de la Figura 3, se muestra el desarrollo de la metodología por medio de un algoritmo para identificar las vertientes de esta metodología, en la aplicación del AMFE en máquinas herramientas de un taller de mecánica ubicado en una institución académica.

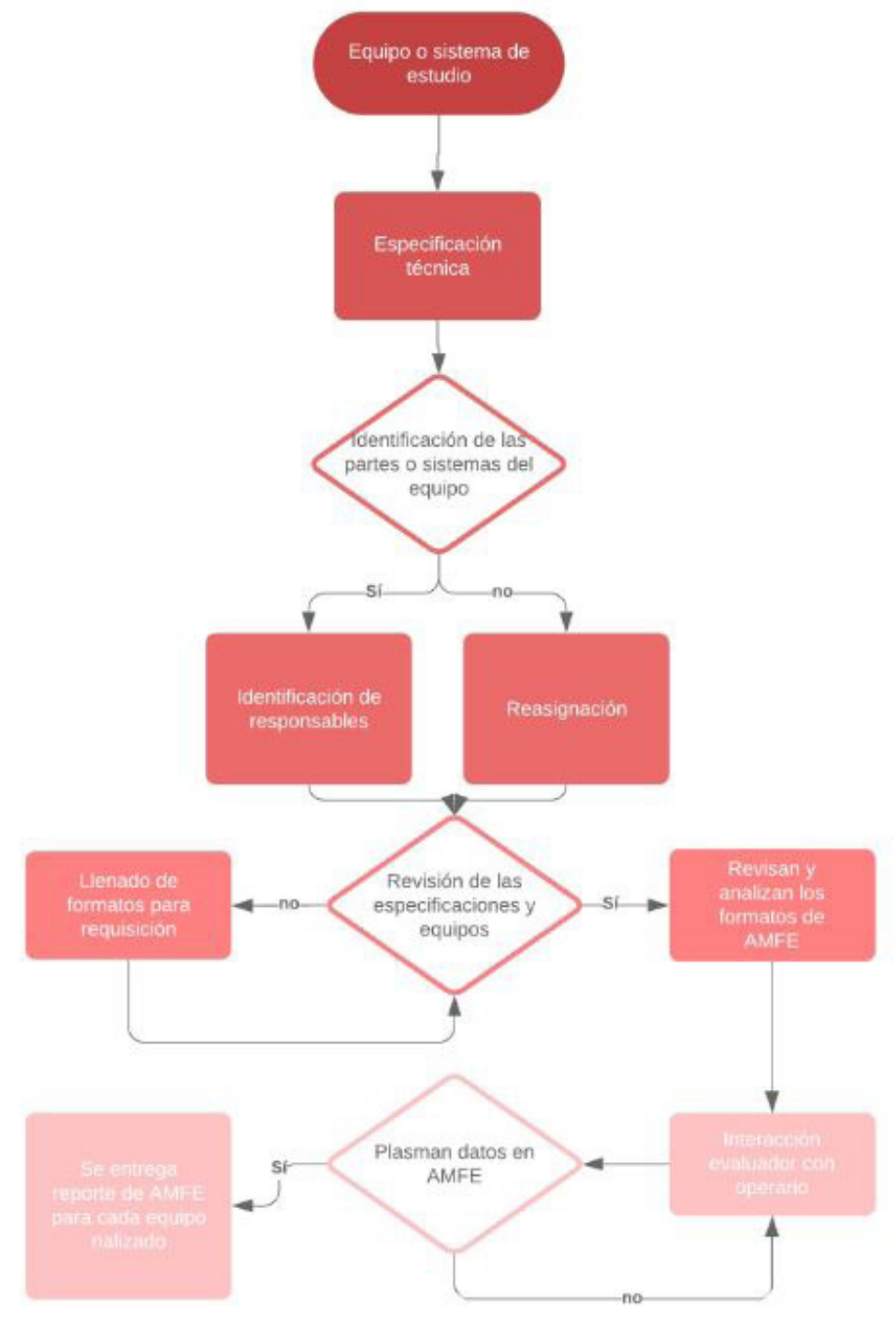

Figura 3. Diagrama de flujo para la aplicación de metodología AMFE

El formato para la aplicación del AMFE se muestra en la Figura 4. Se consideró diferentes campos de aplicación para extrapolar esta metodología al sector industrial. 


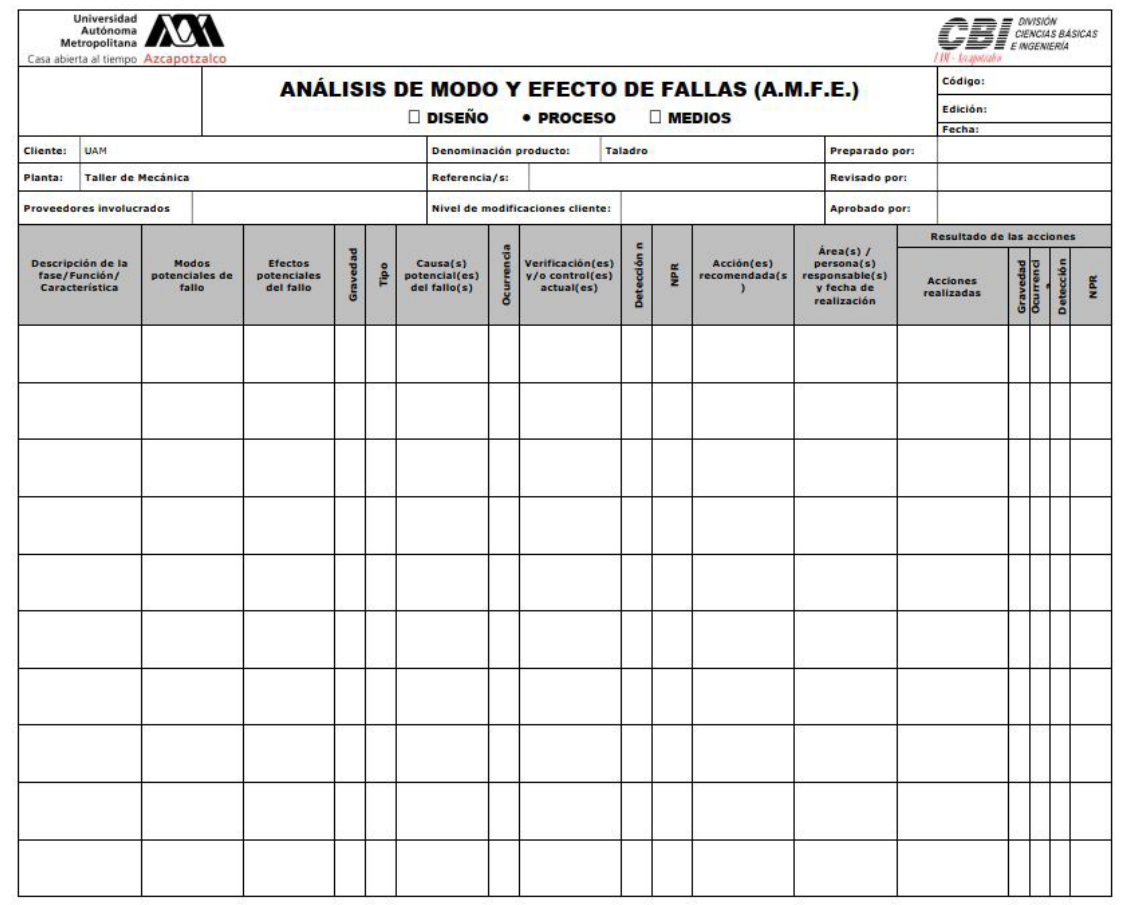

Figura 4. Formato de AMFE para su aplicación en el taller de mecánica

\section{Caso 1. Torno}

Selección del equipo de estudio: se eligió el torno, máquina herramienta que produce trabajo rotativo sobre dos ejes en donde se logran trabajar piezas de diferente geometría, dependiendo del producto a fabricar.

Especificación técnica: obtención de los datos del equipo, en donde se especificaron los parámetros controlables de la máquina y sus límites, se muestran en la Tabla 1.

Tabla 1. Especificación técnica del torno.

\begin{tabular}{|l|l|}
\hline \multicolumn{2}{|c|}{ Especificación Técnica } \\
\hline Volteo sobre la bancada & $0.35 \mathrm{~m}$ \\
\hline Volteo sobre el carro & $1.016 \mathrm{~m}$ \\
\hline Bancada templada y rectificada & $0.254 \mathrm{~m}$ ancho \\
\hline Volteo sobre carro transversal & $0.2032-0.00635 \mathrm{~m}$ \\
\hline Volteo en escote & $0.508 \mathrm{~m}$ \\
\hline Husillo principal & Camlock No. 4 \\
\hline Gama de 12 velocidades & $40-1800$ RPM \\
\hline Gama de roscas sistema inglés y métrico & Aplica \\
\hline Motor principal & $2.2371 \mathrm{~kW}$ \\
\hline Peso neto & $1.2 \mathrm{Tn}$ \\
\hline
\end{tabular}

Identificación de las partes: el torno está compuesto de una bancada, husillos, carro transversal, carro auxiliar, carro longitudinal, manivelas, plato, torreta, caja de transmisión, 
contrapunto.

Identificación de los responsables: técnicos asignados de acuerdo al turno de horario, generalmente dos personas responsables (técnicos).

Aplicación del AMFE: para esta etapa se utiliza un formato general adaptado para la aplicación del AMFE en los equipos propuestos, torno.

\section{Caso 2. Fresadora}

Selección del equipo de estudio: se elige una fresadora vertical, máquina herramienta que produce trabajo rotativo y desplazamiento en tres ejes para obtener las piezas deseadas en la fabricación.

Especificación técnica: obtención de los datos del equipo, en donde se especifican los parámetros controlables de la máquina y sus límites, se muestran en la Tabla 2.

Tabla 2. Especificación técnica de una fresadora vertical.

\begin{tabular}{|l|l|}
\hline \multicolumn{2}{|c|}{ Especificación Técnica } \\
\hline Mesa de trabajo & $0.25 \times 0.90 \mathrm{~m}$ \\
\hline Ranura T & $0.003 \times 0.014 \times 0.054 \mathrm{~m}$ \\
\hline Recorrido longitudinal x & $0.50 \mathrm{~m}$ \\
\hline Recorrido transversal y & $0.165 \mathrm{~m}$ \\
\hline Recorrido vertical z & $0.30 \mathrm{~m}$ \\
\hline Máx. ángulo de rotación de la mesa & $45^{\circ}$ \\
\hline Cono de husillo & $1 \mathrm{SO} 30$ \\
\hline Diámetro del árbol porta fresa & $0.022 \mathrm{y} 0.027 \mathrm{~m}$ \\
\hline Distancia husillo-carenero & $0.160 \mathrm{~m}$ \\
\hline Distancia husillo-mesa & $0.060-0.360 \mathrm{~m}$ \\
\hline Velocidad de husillo, pasos $(\mathrm{r} / \mathrm{min})$ & $12(32-1250)$ \\
\hline Avance longitudinal x pasos $(\mathrm{mm} / \mathrm{min})$ & $12(5.6-181)$ \\
\hline Avance longitudinal y pasos $(\mathrm{mm} / \mathrm{min})$ & $11.3-366$ \\
\hline Avance longitudinal z pasos $(\mathrm{mm} / \mathrm{min})$ & $4.5-146.2$ \\
\hline Motor & $2.4 \mathrm{~kW}$ \\
\hline Dimensiones & $1.40 \times 1.33 \times 1.478 \mathrm{~m}$ \\
\hline Peso & $1300 \mathrm{~kg}$ \\
\hline
\end{tabular}

Identificación de las partes: la fresadora vertical se compone de los siguientes elementos, cabeza, husillo, motor, manivelas para husillo, columna, volantes de movimiento longitudinal y transversal, palancas y caja del sistema.

Identificación de los responsables: técnicos asignados de acuerdo al turno de horario, generalmente dos personas responsables (técnicos).

Aplicación del AMFE: para esta etapa se utiliza un formato general adaptado para la aplicación del AMFE en los equipos propuestos, fresadora vertical.

\section{Caso 3. Taladro vertical}

Selección del equipo de estudio: se elige el taladro industrial como un equipo común en un taller de máquinas herramientas en donde se realizan operaciones de taladrado, abocardado 
y barrenado en diferentes posiciones con herramientas adecuadas.

Especificación técnica: obtención de los datos del equipo, en donde se especifican los parámetros controlables de la máquina y sus límites, se muestran en la Tabla 3.

Tabla 3. Especificación técnica de un taladro industrial.

\begin{tabular}{|l|l|}
\hline \multicolumn{2}{|c|}{ Especificación Técnica } \\
\hline Capacidad máxima de taladro en acero de $70 \mathrm{~kg}$ & $0.040 \mathrm{~m}$ de diámetro \\
\hline Capacidad máxima de taladro en fundición $200 \mathrm{Br}$ & $0.044 \mathrm{~m}$ de diámetro \\
\hline Cono morse & 4 \\
\hline Profundidad máxima de taladrado & $0.015 \mathrm{~m}$ \\
\hline Avance de taladro & Manual \\
\hline Potencia del motor de 2 velocidades & $1.8 / 3.2 \mathrm{CV}$ \\
\hline Transmisión & $\begin{array}{l}\text { Variador mecánico de } \\
\text { velocidad con poleas }\end{array}$ \\
\hline No. De velocidades & Variable \\
\hline Gama de velocidades motor 750/1500 rpm $50 \mathrm{~Hz} 3 \mathrm{Ph}$. & $80-1170 \mathrm{rpm}$ \\
\hline Dimensiones de la mesa & $0.40 \times 0.42 \mathrm{~m}$ \\
\hline Diámetro de la columna & $0.125 \mathrm{~m}$ \\
\hline Distancia del centro husillo a la columna & $0.30 \mathrm{~m}$ \\
\hline Distancia husillo -mesa & Min. 0.15 / Máx. $0.95 \mathrm{~m}$ \\
\hline Distancia husillo-base & $1.310 \mathrm{~m}$ \\
\hline Peso neto aproximado & $295 \mathrm{~kg}$ \\
\hline
\end{tabular}

Identificación de las partes: el taladro industrial está compuesto de los siguientes elementos, mesa, columna-soporte, cabezal fijo, sistema de fijación, ménsulas, eje principal, volante de avance y plato giratorio.

Identificación de los responsables: técnicos asignados de acuerdo al turno de horario, generalmente dos personas responsables (técnicos).

Aplicación del AMFE: para esta etapa se utiliza un formato general adaptado para la aplicación del AMFE en los equipos propuestos, torno industrial.

\section{RESULTADOS}

Se presentan los datos aplicando AMEF, de la siguiente manera.

\section{Caso 1. Torno.}

En este análisis (Figura 5), con respecto a un torno convencional horizontal, se logró observar e identificar que existen mejoras en la reducción de las acciones para optimizar y mejorar las condiciones de un torno para lograr las actividades correspondientes en un período escolar. Se han extrapolándolo condiciones de trabajo para la industria y minimizar los costos de mantenimiento para el equipo. 


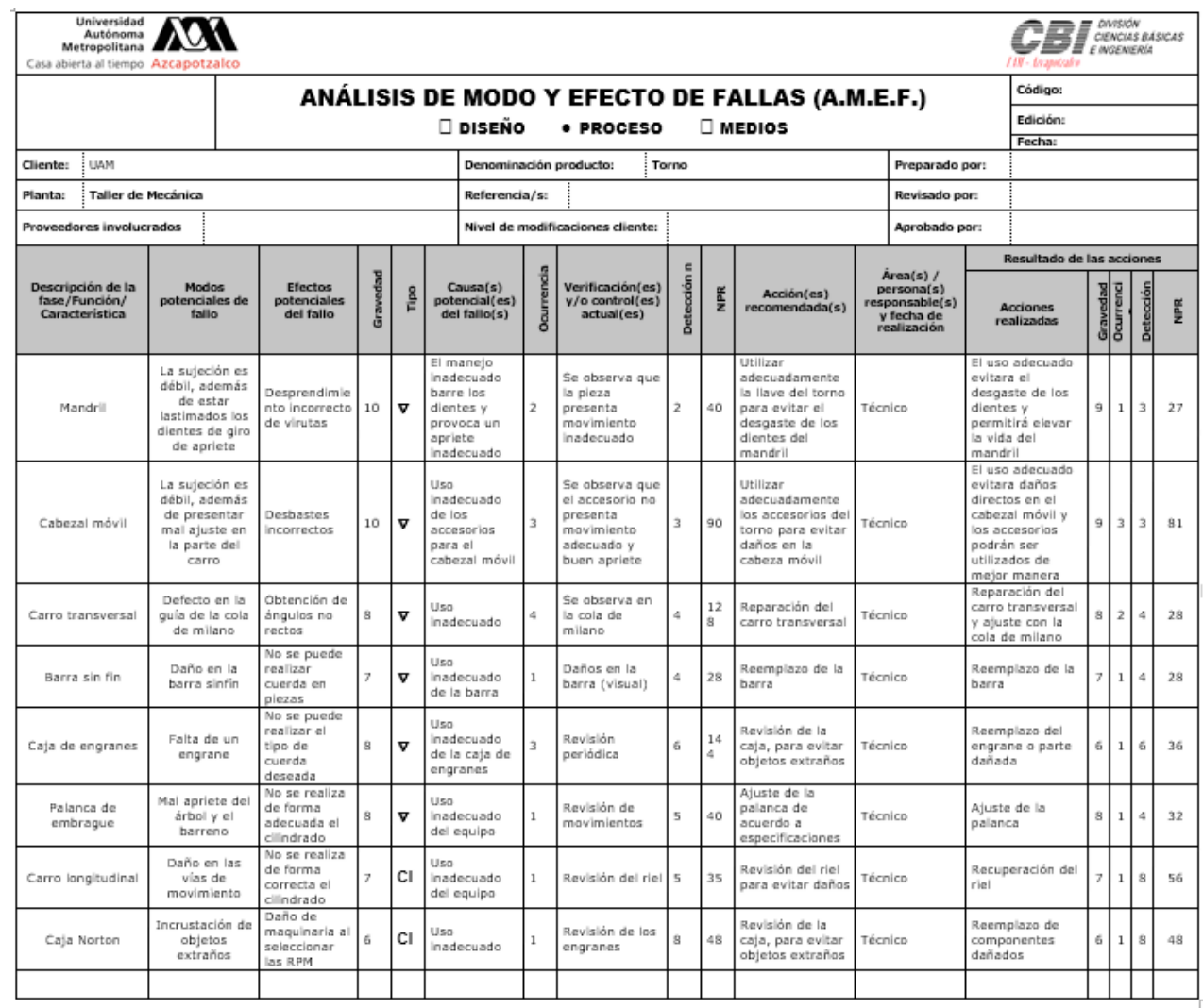

Figura 5. AMFE para el caso de un torno

En la Tabla 4 y Figura 6, se observa la situación crítica analizada por medio de un AMFE aplicado a un torno, en donde es claro la reducción del NPR (2), después de realizar los ajustes para un mejor mantenimiento en el equipo y producción, al igual que centrarse en establecer condiciones de mantenimiento en los tres aspectos, predictivo, preventivo y correctivo, de la caja de engranes, cuyo elemento es el más afectado y por ello direccionar la atención en futuros análisis. Posteriormente el estudio de otro AMFE para el carro transversal y cabeza móvil, conjugando las actividades de mantenimiento con los operadores de máquina, en conjunto con la parte administrativa de mantenimiento y los mecánicos para desarrollar las acciones previstas en la mejora del proceso de mantenimiento en la implementación. 
Tabla 4. Resultados del AMFE-Torno

\begin{tabular}{|l|c|c|}
\hline \multicolumn{1}{|c|}{ Torno } & NPR (1) & NPR (2) \\
\hline Mandril & 40 & 27 \\
\hline Cabezal móvil & 90 & 81 \\
\hline Carro transversal & 128 & 64 \\
\hline Barra sin fin & 28 & 28 \\
\hline Caja de engranes & 144 & 36 \\
\hline Palanca de embrague & 40 & 32 \\
\hline Carro longitudinal & 35 & 56 \\
\hline Caja Norton & 48 & 48 \\
\hline
\end{tabular}

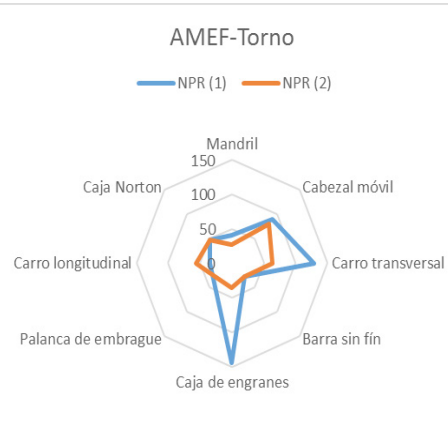

Figura 6. AMFE-Torno

\section{Caso 2. Fresadora}

El número de prioridad de riesgos (NPR) tiene un valor menor, de acuerdo con los ajustes establecidos para la fresadora vertical, detectando la importancia del mantenimiento en cada una de sus etapas, que específicamente en este caso se trabaja, de acuerdo con el análisis, en mantenimiento preventivo y predictivo para lograr el máximo tiempo de trabajo para la maquinaria que cumple con estas características. (Figura 7). Sin embargo, se debe rescatar que la aplicación del AMFE, deja gran experiencia y atención en los sectores principales de una fresadora, permitiéndole aumentar el ciclo de producción. 


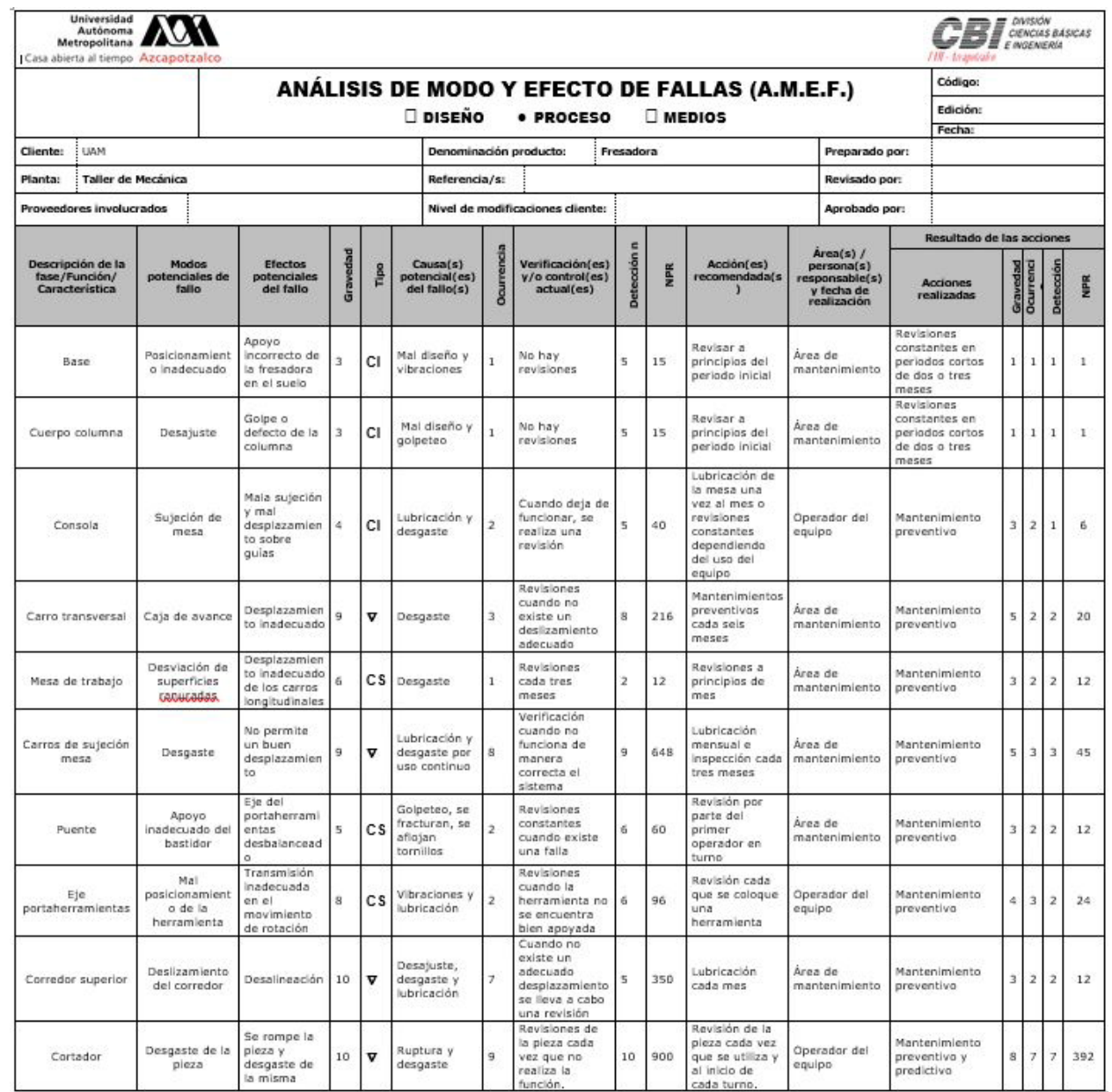

Figura 7. AMFE de una fresadora vertical

Para el caso de la fresadora, se identificó por medio de la dimensión de las áreas, aquella sección o elemento que es considerado crítico en cuanto al proceso de mantenimiento, con lo cual se abordarían los parámetros a controlar en este análisis y favorecer el funcionamiento y los procesos de producción para una fresadora de estas características (Figura 8).

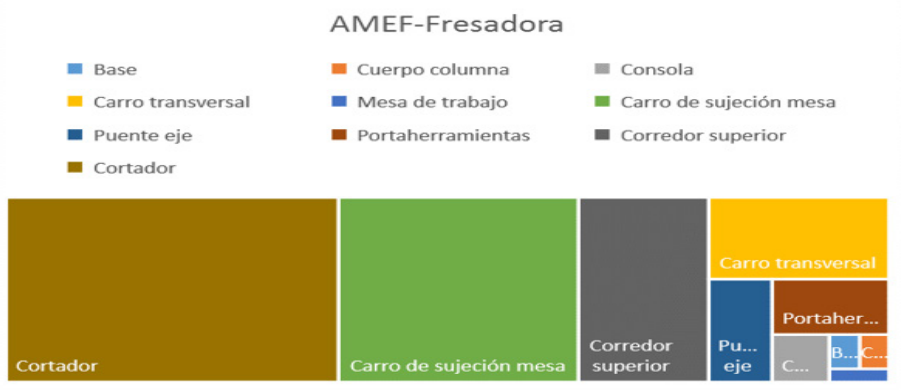

Figura 8. AMFE-Fresadora 
En la Tabla 5, se muestra la mejora existente y radical para el carro de sujeción de mesa y el cortador, los cuales obtuvieron un NPR (1) alto y posterior al análisis las mejoras expuestas reducen de tal manera que permite reestructurar en otro momento el AMFE para seguir obteniendo mejores resultados en lo correspondiente a las partes críticas en el análisis, como lo es el carro de sujeción de mesa, cortador, corredor superior y carro transversal.

A diferencia que el torno convencional, se encontró que la fresadora ofrece una amplia gama de análisis en cada una de sus partes por la complejidad que existe en el equipo como un ente de la manufactura.

De la misma forma se logra identificar los parámetros críticos en las fallas y sus posibles tendencias enfocadas en la multidisciplinariedad para formular la solución adecuada en conjunto con los interesados de producción y mantenimiento.

Tabla 5. Resultados del AMFE-Fresadora

\begin{tabular}{|l|c|c|}
\hline \multicolumn{1}{|c|}{ Fresadora } & NPR (1) & NPR (2) \\
\hline Base & 15 & 1 \\
\hline Cuerpo columna & 15 & 1 \\
\hline Consola & 40 & 6 \\
\hline Carro transversal & 216 & 20 \\
\hline Mesa de trabajo & 12 & 12 \\
\hline Carro de sujeción mesa & 648 & 45 \\
\hline Puente eje & 96 & 24 \\
\hline Portaherramientas & 96 & 24 \\
\hline Corredor superior & 350 & 12 \\
\hline Cortador & 900 & 392 \\
\hline
\end{tabular}

\section{Caso 3. Taladro Industrial}

En la Figura 9, se observa que las etapas evaluadas son pocas representando lo esencial del equipo para su evaluación con el AMFE, partiendo de ello lograr reducir el NPR, aumentando las condiciones estándar de trabajo para el taladro, mostrando una mejora para el caso del mandril, que es una de las partes esenciales en cualquier proceso de taladrado. 


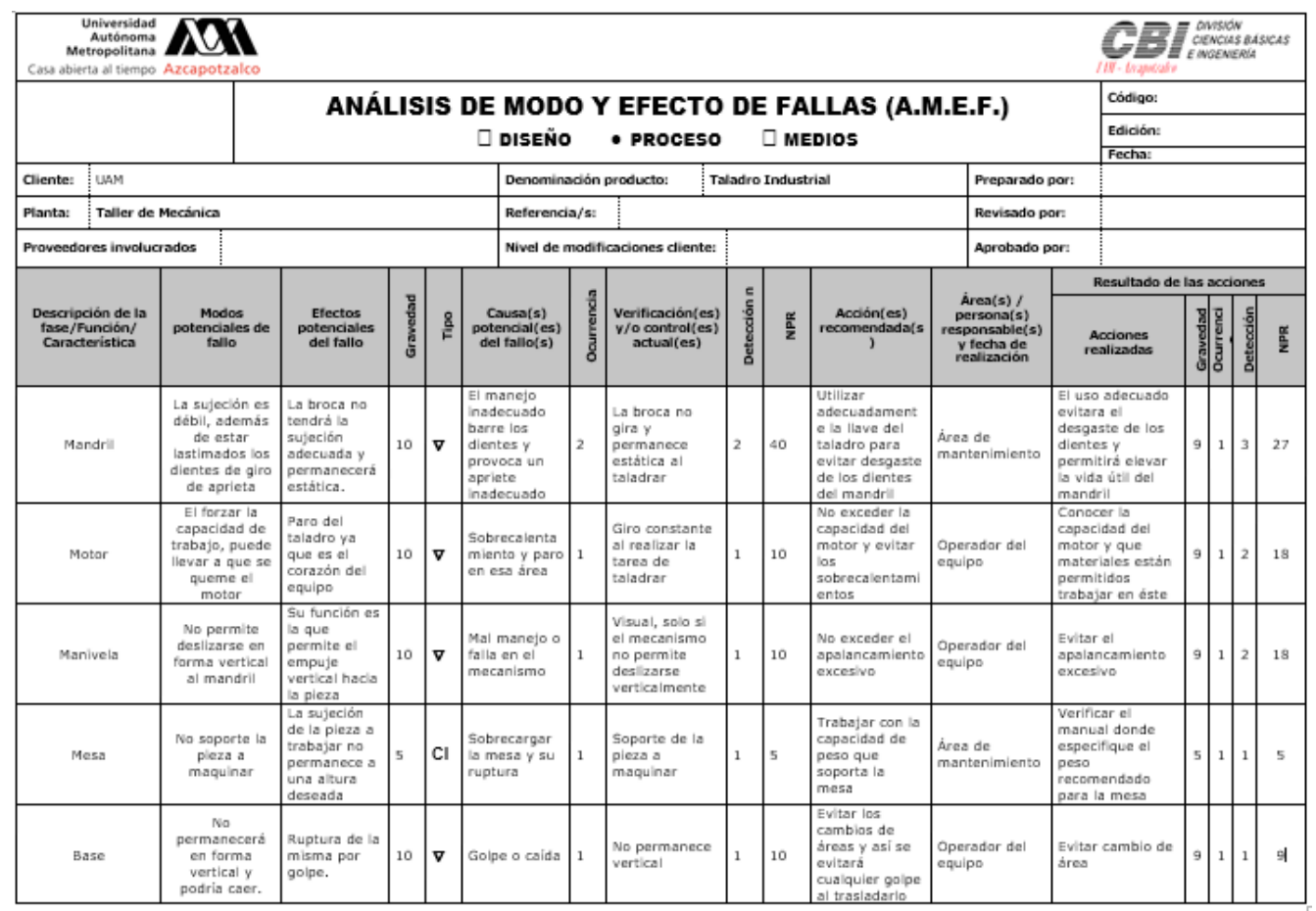

Figura 9. AMFE de un taladro Industrial

Con respecto al caso de un taladro industrial, existen pocos elementos de análisis, pero no lo excluye de abordarse con el AMFE y observar (Tabla 6 y Figura 10) cuales son las tendencias de estos equipos dentro de un plan de mantenimiento y los resultados que este ofrece, para este caso el mandril es el aspecto crítico que debe involucrarse en análisis cíclicos para incentivar nuevas propuestas en los estudios de taladros industriales. Algo que requiere de atención, es el motor y la manivela, ya que después del análisis se observa que el NPR(2) en lugar de disminuir aumento y ello indica que lo más probable es realizar nuevamente otro AMFE con mayor cantidad de criterios a evaluar como parte del proceso cíclico en la determinación de errores o fallas que presenta el sistema o en su defecto analizar la sección de mejora para identificar los puntos de bifurcación y su análisis, cuyos puntos se encuentran ubicados en las secciones de unión en el taladro, como lo es la banda, sistema de engranajes y bancada, que son elementos sometidos a vibraciones que provocan la inestabilidad en el sistema. 
Tabla 6. Resultados del AMEF-Taladro

\begin{tabular}{|c|c|c|}
\hline Taladro & NPR (1) & NPR (2) \\
\hline Mandril & 40 & 27 \\
\hline Motor & 10 & 18 \\
\hline Manivela & 10 & 18 \\
\hline Mesa & 5 & 5 \\
\hline Base & 10 & 9 \\
\hline
\end{tabular}

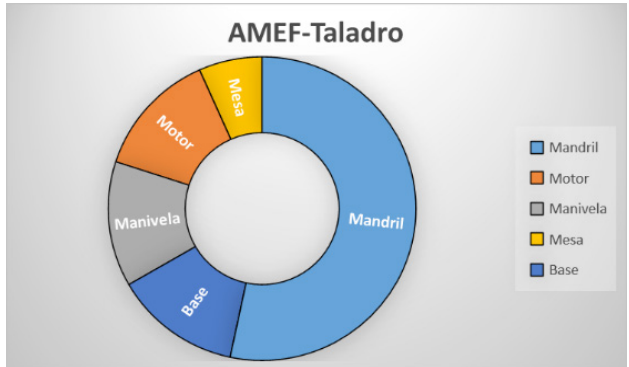

Figura 10. AMFE-Taladro

\section{CONCLUSIÓN}

Después de aplicar el análisis de modo y efecto de fallas (AMFE) en un taller de ingeniería mecánica en donde se trabajan diversos sistemas que están compuestos por equipos, tornos, taladros, fresadoras, esmeriles, entre otros, se logra identificar la importancia de aplicar herramientas diferentes para los procesos y procedimientos del mantenimiento como parte de la gestión y los impactos que ofrece cuando se aplica de manera adecuada, con respecto a la experiencia adquirida y la que se tiene al momento de llevar a cabo el proceso.

Al aplicar el AMFE a los tres casos expuestos, se perciben las tendencias del mantenimiento, que se encuentran relacionadas de forma directa con dos factores, uno es la optimización del proceso de mantenimiento que se logró aplicando un AMFE, cuyo objetivo se basa en aumentar de manera considerable la inversión en dicha sección y por otro lado concientizando a todo el personal del taller de maquinado para la implementación de un programa de mantenimiento con los resultados de la herramienta, mostrando avances significativos para que los ambientes en ese ámbito ofrezcan crecimientos significativos y lograr extrapolarlos a otras entidades que mantengan la idea de mejora continua a través de un proceso de mantenimiento.

Por medio del AMFE, se identificaron los elementos críticos que ofrecen áreas de oportunidad para el mantenimiento en sus etapas preventivas y correctivas de los equipos analizados, logrando una relación directa de los sistemas con los encargados de mantenimiento dentro del taller de manufactura. 


\section{REFERENCIAS}

CARTIN-ROJAS, A., VILLARREAL-TELLO, A y MORERA, A. (2014). Implementación del análisis de riesgo en la industria alimentaria mediante la metodología AMEF: enfoque práctico y conceptual. Rev. Med. Vet. [en línea]. 2014(27), 133-148. Disponible en: http://www.scielo. org.co/scielo.php?script=sci_arttext\&pid=S0122-93542014000100012\&lng=en\&nrm=iso

CONSUEGRA MATEUS, O. (2015). Metodología AMFE como herramienta de gestión de riesgo en un hospital universitario. Cuadernos Latinoamericanos de Administración [en línea]. 21(20), 37-49. Disponible en: https://www.redalyc.org/articulo.oa?id=409640743004

FORNÉS-RIVERA, R., OCHOA-ESPINOZA, L., CANO-CARRASCO, A y GONZÁLEZVALENZUELA, E. (2016). Gestión de Mantenimiento Centrado en Confiabilidad en el área de laboratorios de una Institución de Educación Superior. Revista de Aplicaciones de la Ingeniería [en línea]. 3(8): 77-86. Disponible en: http://www.ecorfan.org/bolivia/researchjournals/ Aplicaciones_de_la_Ingenieria/vol3num8/Revista_Aplicaciones_de_la_Ingenieria_V3_ N8_10.pdf

GARCIA, J., SANTANA, Z., ZUMALACÁRREGUI, L., QUINTANA, M., MILA, L., RAMOS, M., BELDARRÁN, A. (2012). Aplicación del análisis de riesgo a la producción de proteínas recombinantes expresadas en Escherichia coli. Revista Vaccimonitor, 21(2), [Mayo-Agosto], pp. 35-42. Disponible en: http://www.redalyc.org/articulo.oa?id=203424333007

GONZÁLEZ, J.C., MYER, R.A. y PACH+ON-MUÑOZ, W. (2017). La evaluación de los riesgos antrópicos en la seguridad corporativa: del Análisis Modal de Fallos y Efectos (AMFE) a un modelo de evaluación integral del riesgo. Revista Científica General José María Cordova, 15(19)[Enero-Junio]. pp. 269-289. Disponible en: https://www.redalyc.org/articulo. oa?id=476255361012

HERNANDEZ-GOMEZ, A., ESCOBAR-TOLEDO, C., LARIOS-PRADO, J M. y NORIEGAMORALES, S. (2015). Factores críticos de éxito para el despliegue del mantenimiento productivo total en plantas de la industria maquiladora para la exportación en Ciudad Juárez: una solución factorial. Contad. Adm [en línea]. 60, (suppl.1), 82-106. Disponible en: http:// dx.doi.org/10.1016/j.cya.2015.08.005.

HERRERA-GALÁN, M. (2017). Aplicación de la gestión de riesgo a equipos y sistemas productivos. Dyna [en línea] 84(Jul-Sep). Disponible en: http://www.redalyc.org/articulo. oa? id $=49655539028$

NAPOLES-VILLA, A., SILVA-RICARDO, Y., MARRERO-FORMARIS, C. (2016). Análisis Modal de Fallo y sus Efectos de las Normas de Competencias. Revista Ciencias Holguín [en línea]. 22(2) [Abril-Junio], pp. 1-15. Disponible en: http://www.redalyc.org/articulo. oa? id=181545579001

ORTIZ-USECHE, A., RODRÍGUEZ-MONROY, C. e IZQUIERDO, H. (2013). Gestión de mantenimiento en pymes industriales. Revista Venezolana de Gerencia [en línea]. 18 (EneroMarzo). Disponible en:http://www.redalyc.org/articulo.oa?id=29026161004 ISSN 
PINEDA-ZAPATA, U., ESTRADA-HERNÁNDEZ, M. y RODRÍGUEZ-MARRUGO, I. (2013). Ingeniería de la calidad aplicada a la planificación de un servicio. Revista Virtual Universidad Católica del Norte [en línea].38(febrero-mayo), 206-224. Disponible en: http://www.redalyc. org/articulo.oa?id=194225730016

ROSALES-AMBRIZ, J.L., TAPIA-BRISEÑO, J.A., ÁVILA-PEDROZA, S.A., CEJA-CAZARES, H.R., CARRILLO-GUTIERREZ, T. y ARREDONDO-SOTO, K.C. (2015). Propuesta de plan de mantenimiento preventivo de los vehículos de transporte en una empresa pública de servicios de agua. Revista Aristas: Investigación Básica y Aplicada [en línea]. 3(6), 35-42. Disponible en: http://fcqi.tij.uabc.mx/usuarios/revistaaristas/numeros/N6/N6.pdf

VÁZQUEZ-VALENCIA, A., SANTIAGP-SÁEZ, A., PEREA-PÉREZ, B., LABAJO-GONZÁLEZ, E. y ALBARRÁN-JUAN, M.E. (2018). Utilidad de análisis modal de fallos y efectos para mejorar la seguridad en la movilización del paciente crítico intubado. Revista Colombiana de Anestesiología. 46(1), pp. 3-10. Disponible en: http://www.scielo.org.co/pdf/rca/v46n1/ es_0120-3347-rca-46-01-00003.pdf 
ISSN 0717-9103

ISSN Online 0718-8307

Universidad del Bío-Bío 